\title{
Diastolic Aorto-Right-Atrial Fistulation in Aortic and Tricuspid Valve Endocarditis
}

\author{
Lukas Frey $^{1}$ Christoph Starck ${ }^{1}$ Volkmar Falk ${ }^{1} \quad$ Simon Sündermann ${ }^{1}$ \\ ${ }^{1}$ Department of Cardiovascular Surgery, University Hospital Zurich, \\ Zurich, Switzerland

\begin{abstract}
Address for correspondence Simon Sündermann, MD, Department of Cardiovascular Surgery, University Hospital Zurich, Rämistrasse 100, Zürich 8091, Switzerland (e-mail: simon.suendermann@usz.ch).
\end{abstract}

Thorac Cardiovasc Surg Rep 2014;3:19-22.

\begin{abstract}
Background Aorto-right-atrial fistula in native valve endocarditis is very rare. Case Description A 45-year-old woman was referred with an endocarditis with a perforated right cusp of the aortic valve with at least moderate insufficiency and an

Keywords affected tricuspid annulus with vegetations. In addition to this, an aorto-cavitary fistula

- aortic valve and root from the aortic sinus to the right atrium with a holodiastolic left-right shunt had been

- endocarditis

- cardiovascular surgery detected. Streptococci viridans were found as underlying pathogen. Complete replacement of the aortic root and resection of the fistula were performed with good result. Conclusion Endocarditis with fistula formation is rare and has to be treated aggressively.
\end{abstract}

\section{Introduction}

A fistulous tract formation from the aortic sinus to the right atrium due to an infective endocarditis (IE) is unusual and affords uncommon treatment. Here, we describe the case of a 45 -year-old woman suffering from such a constellation.

\section{Case Presentation}

A 45-year-old woman with a history of anemia and bronchiectasis in her left upper lobe was referred to the clinic of cardiology due to palpitations and malaise. Since 4 weeks she had suffered from fatigue, weight loss, and elevated temperatures.

Physical examination revealed irregular tachycardia (heart rate, $118 / \mathrm{min}$ ), hypotension ( $95 / 55 \mathrm{~mm} \mathrm{Hg}$ ), a body temperature of $38.6^{\circ} \mathrm{C}$, and a new diastolic murmur (3/6). Electrocardiogram revealed a narrow complex tachycardia with changing QRS amplitude and irregular P-wave. Except for an elevated CRP level, all other laboratory values were without pathological findings.

Because of the new diastolic murmur, a transthoracic echocardiography and a transesophageal echocardiography were conducted and confirmed the clinical suspicion of IE with visualization of a perforated right coronary cusp of the aortic valve and an affected tricuspid annulus with vegeta-

received

January 6, 2014

accepted

April 8, 2014

published online

July 24, 2014 tions. The tricuspid aortic valve showed moderate to severe eccentric insufficiency. In addition to this, an aorto-cavitary fistula (ACF) from the aortic sinus to the right atrium with a holodiastolic left-right shunt had been detected by Doppler echocardiography (-Fig. 1). The cusp was supposed to block the fistula ostium during systole. The right ventricle showed a moderate tricuspid insufficiency with no evidence of hypertrophy but a slightly reduced ejection fraction (biplane $=52 \%$ ). The left ventricle was normal in size and systolic function. Coronary artery disease was ruled out in coronary angiography.

Blood cultures of $2 \times 2$ were obtained in the beginning and all of them were tested positive for Streptococci viridans susceptible for penicillin and gentamicin. For exclusion of cerebral septic emboli, a brain computed tomography was performed without any pathological findings. In the thoracic computed tomography performed during the same investigation, the fistula could be confirmed (-Fig. 2).

\section{Treatment}

An empiric antibiotic therapy with penicillin, gentamicin, and vancomycin in accordance with the national guidelines for the treatment of IE had been undertaken initially. The
License terms

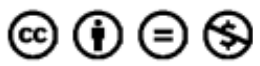

(c) 2014 Georg Thieme Verlag KG
Stuttgart · New York 10.1055/s-0034-1381746. ISSN 2194-7635. 


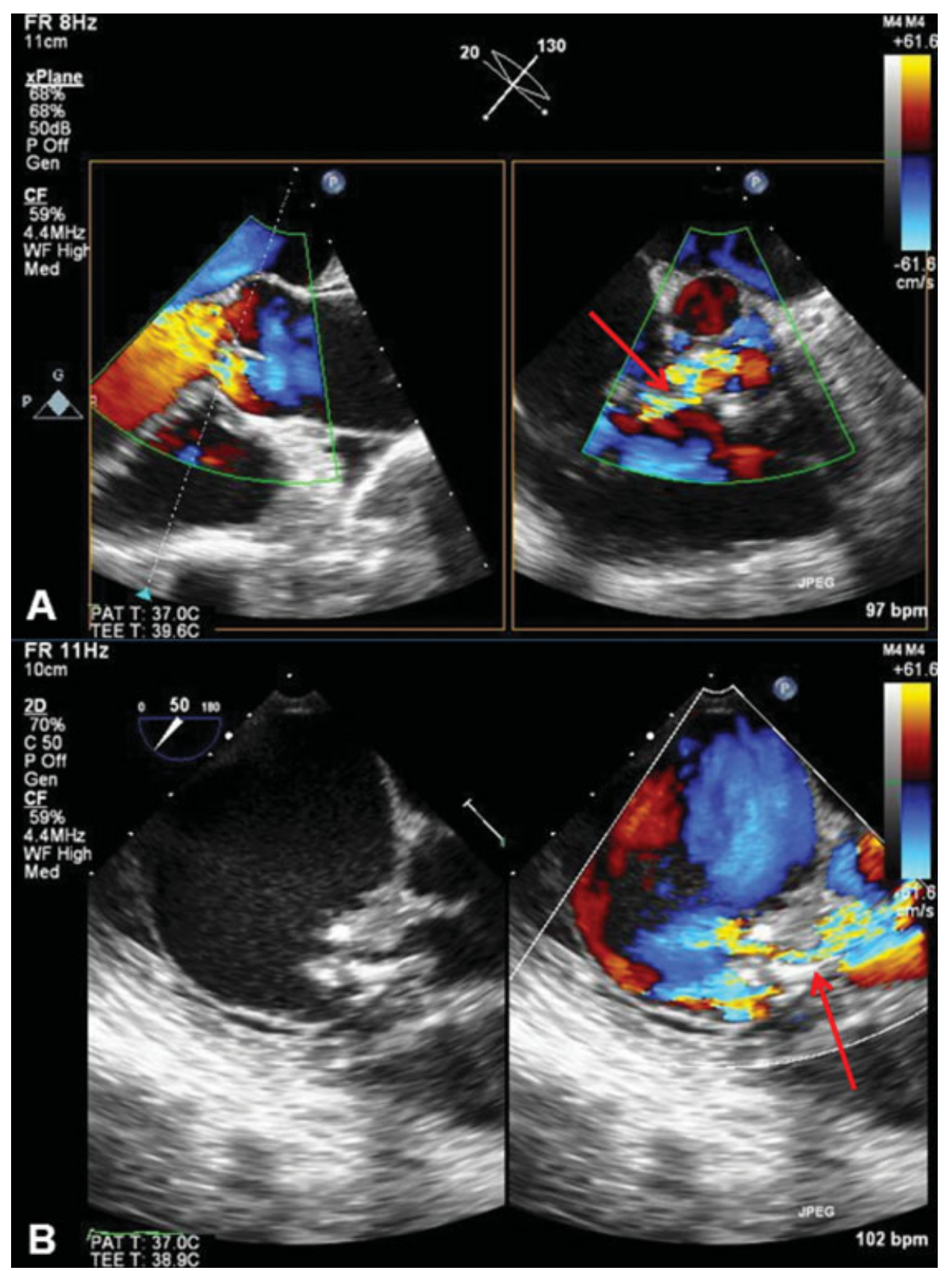

Fig. 1 Preoperative transesophageal echocardiography. (A) X-plan long axis (left) and short axis (right) with color Doppler signal. (B) Echocardiographic view of the fistula with and without color Doppler signal. In both images, the fistula is marked with a red arrow.

patient was scheduled for urgent surgery. Installation of cardiopulmonary bypass was performed in the standard fashion with cannulation of the ascending aorta and both caval veins. Bretschneider cardioplegia was administered antegrade into the coronary ostia. Inspection of the operative situs showed a perforation with a diameter of around $1 \mathrm{~cm}$ and vegetation on the margin of the right coronary cusp. Below the right coronary ostium, a fistula could be identified connecting the aorta directly with the right atrium. By accessing the right atrium, the end of the fistula was located close to the septal area of the tricuspid annulus (-Fig. 3 ). After the excision of the aortic root, radical debridement of the fistula was possible. This was followed by a reconstruction of the right atrium and aortic root with pericardium patches to finally enable the implantation of a $23-\mathrm{mm}$ biological composite graft (Freestyle Aortic, Freestyle, Med- tronic Inc., Minneapolis, United States). The moderate tricuspid valve insufficiency was accepted to avoid further implantation of foreign material in the setting of an acute endocarditis.

Material for further microbiological analysis was collected. After the reperfusion of the heart, sinus rhythm was present with intermittent conduction block. Thus, implantation of an epicardial pacemaker probe was performed for a potential subsequent need of a pacemaker.

On postoperative day (POD) $1, \mathrm{X}$-ray image illustrated a right-sided hemothorax which required a rethoracotomy and evacuation of a hematoma.

PCR investigation after surgery proved Streptococcus mitis vegetation. Vancomycin was stopped and penicillin and gentamicin were continued for 6 weeks and 14 days, respectively. The patient was discharged at POD 15. 


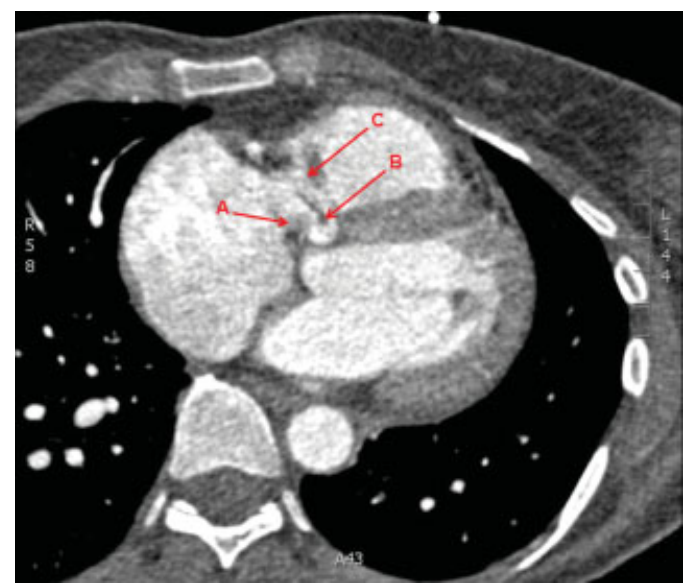

Fig. 2 Preoperative computed tomography. (A) Aortic annulus; (B) subaortic abscess and fistula; (C) tricuspid valve.

During the rehabilitation, the patient complained about dyspnea, vertigo, thoracic pressure sensation, and intermittent palpitations. Echocardiography revealed a shunt from the left outflow tract to the right atrium due to a ventricular septal defect (VSD) located in the pars membranacea. The biological composite graft was evaluated to be in correct position with normal valvular function without any evidence of insufficiency. The finding of new VSD indicated a surgical correction, which was performed 2 months (POD 54) after the first cardiac intervention. Intraoperatively, the initially implanted patch was detached. The closure was performed by suturing a new pericardium patch on either side. The following postoperative course was uneventful and the echocardiographic examination showed a successful closure of the shunt.

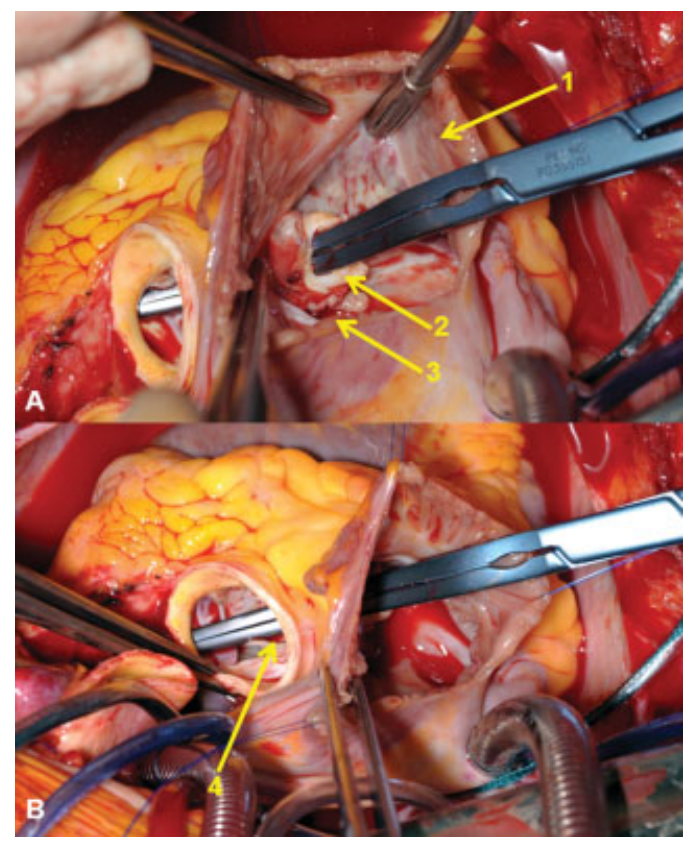

Fig. 3 Intraoperative situs. (A) Right atrial view; (B) aortic view. 1: right atrium; 2: orifice of the fistula in the right atrium; 3 : annulus of the tricuspid valve; 4 : orifice in the right coronary cusp of the aortic valve.
Due to persisting postoperative third-degree atrioventricular block, the implantation of a dual chamber pacemaker was necessary 7 days later.

Four months later, the patient was readmitted for removal of her sternal wires. At that time, she presented asymptomatic and was discharged home without any signs for recurrent endocarditis on a transitional anticoagulation with phenprocoumon, which was switched to aspirin in the further course.

\section{Discussion}

Here, we present the case of an aorto-right-atrial fistula in a case of native valve endocarditis (NVE).

Aorto-atrial fistulae are a rare manifestation of IE. Anguera et al reported a rate of $1.8 \%$ of fistulous formation in patients with NVE and of $3.5 \%$ in prosthetic valve endocarditis. ${ }^{1}$ The most frequent infected site in NVE is the aortic valve (38-55\%) and the mitral valve (39-41\%) followed by the other valves $(6-13 \%){ }^{2}$ For the aortic valve, all three sinuses and the four cardiac chambers have been described as to be equally involved in fistulous tract formation. ${ }^{1}$ In our case, the fistula was found to be connected from the right coronary cusp to the right atrium close to the annulus of the tricuspid valve. This location has not yet been described to our knowledge. Another unique finding in this case was that there was only diastolic left-right shunt, due to the suspected occlusion of the fistula entrance during systole by the aortic valve cusp. Three case descriptions of aorto-right-atrial fistulation are available $^{3-5}$ in the literature. In two of them, the aortic valve was bicuspid and the tricuspid valve was involved only in one case. Detailed treatment is not described in any of the cases.

Viridans streptococci were the underlying pathogen in our case. "Viridans streptococci vary in their invasiveness and abscess potential." 6 Streptococcus mitis is a member of the $S$. viridans group and is found more often in tricuspid valve endocarditis of patients with intravenous drug abuse. ${ }^{7}$ Medical history and clinical presentation were negative concerning intravenous drug abuse in our case.

Early and properly timed operative intervention in combination with optimal antibiotic therapy and elaborate preand postoperative care is the best approach to achieve an acceptable outcome in case of active IE. ${ }^{8}$ In a case of doublesided IE with a fistulous connection and associated extensive perivalvular damage, therapy is even more complicated.

In conclusion, with this case presentation, we tried to show that for patients with ACF in aortic NVE, timely diagnosis, accurate antibiotic therapy, and early, aggressive cardiac surgery are necessary to provide the best possible outcome. A proper postoperative monitoring with electrocardiography, echocardiography, and other diagnostic instruments assure timely detection of potential complications of ACF in IE.

\section{References}

1 Anguera I, Miro JM, Vilacosta I, et al; Aorto-cavitary fistula in Endocarditis Working Group. Aorto-cavitary fistulous tract formation in infective endocarditis: clinical and echocardiographic 
features of 76 cases and risk factors for mortality. Eur Heart J 2005; 26(3):288-297

2 Castonguay MC, Burner KD, Edwards WD, Baddour LM, Maleszewski JJ. Surgical pathology of native valve endocarditis in 310 specimens from 287 patients (1985-2004). Cardiovasc Pathol 2013;22(1):19-27

3 Gunarathne A, Hunt J, Gershlick A. Aorto-right atrial and right ventricular fistulae: a very rare complication of native bicuspid aortic valve endocarditis. Heart 2013;99(22):1708

4 Dias A, Mankodi D, Smith S. Aorta to right atrial fistula presenting as a rare complication of native bicuspid aortic valve endocarditis. J Invasive Cardiol 2013;25(1):61
5 Rocha S, Almeida J, Santos A, Mateus P, Pinho P. Aortic native valve endocarditis complicated with aorto-right atrial fistula [in Portuguese]. Rev Port Cir Cardiotorac Vasc 2008;15(2):75-77

6 Alkhatib B, Schoch PE, Cunha BA. Viridans streptococcal (Streptococcus mitis) biosynthetic aortic prosthetic valve endocarditis (PVE) complicated by complete heart block and paravalvular abscess. Heart Lung 2012;41(6):610-612

7 Rapeport KB, Girón JA, Rosner F. Streptococcus mitis endocarditis. Report of 17 cases. Arch Intern Med 1986;146(12):2361-2363

8 Perić M, Vuk F, Huskić R, et al. Active infective endocarditis: low mortality associated with early surgical treatment. Cardiovasc Surg 2000;8(3):208-213 